\title{
Effet comparé de la dose de lisier de porc utilisé comme fertilisant en étang sur la richesse et la distribution des taxons phytoplanctoniques
}

\author{
NANA Towa Algrient ${ }^{1 *}$, Songmo Berlin ${ }^{1}$, EFOLE Ewoukem Thomas', FONKWA Georges', KOM \\ Meliphe Francis², TCHOUMBOUE 1 \\ 'Laboratoire d'Ichtiologie et Hydrobologie Appliquée, Faculté d'Agronomie et des Sciences Agricoles Université de \\ Dschang, BP 222 Dschang - Cameroun \\ 2Laboratoire de Botanique Appliquée, Faculté des Sciences, Université de Dschang-Cameroun \\ *Corresponding author : algrient@yahoo.fr Tél : +237673180089
}

Original submitted in on $3^{\text {rd }}$ June 2020. Published online at www.m.elewa.org/journals/ on $31^{\text {st }}$ August 2020

https://doi.org/10.35759/JABs.152.3

\section{RESUME}

Objectif : La pisciculture en étang se caractérise par une grande diversité de pratiques piscicoles des plus extensives aux plus intensives en passant par la pratique semi-intensive basée sur la fertilisation. Ainsi, le présent travail portant sur la culture du phytoplancton a été initié en vue de contribuer à l'amélioration de la production de poisson en étangs fertilisés à travers une meilleure gestion des fertilisants.

Méthodologie et résultats : L'essai a été conduit dans 9 étangs en dérivation de même superficie $(5,7 \times 5,7$ $\mathrm{m}$ ) et de profondeur $1 \mathrm{~m}$ à la station aquacole de la Ferme d'Application et de Recherche (F.A.R) de l'Université de Dschang. Un filet de 1,5 mm d'ouverture de maille a été fixé sur le tuyau d'alimentation de chaque étang en vue d'éviter l'intrusion des poissons venant de la retenue. Chacun des traitements constitués de $0 ; 800$ et $1000 \mathrm{~kg} / \mathrm{ha}$ de lisier a été appliqué dans 3 étangs de façon aléatoire. Les résultats ont montré que la richesse des espèces ainsi que des genres phytoplanctoniques a diminué avec l'apport du lisier, soit la valeur 164 espèces enregistrées dans les étangs non fertilisés contre 124 dans le traitement à la dose de $800 \mathrm{~kg} / \mathrm{ha}$ de lisier. Les Cyanophytes et Bacillariophytes ont été le plus abondant dans les étangs non fertilisés alors que les Euglenophytes et les Pyrrophytes ont été dominant dans les étangs fertilisés à la dose de $1000 \mathrm{~kg}$. La densité phytoplanctonique a augmenté avec l'accroissement de la quantité de lisier en étang.

Conclusion et application : La dose de lisier de porc a influencé significativement la richesse et la densité phytoplanctonique en étang. En somme la dose $1000 \mathrm{~kg}$ peut déjà être utilisée sous réserve de rechercher la dose optimale de production phytoplanctonique en étang au regard de l'évolution progressive de la densité phytoplanctonique avec l'augmentation de la dose. Ces données vont permettre à améliorer l'élevage des espèces de poisson phytophage notamment les carpes en étang.

Mots clés : Fertilisation, lisier de porc, taxon, phytoplancton. 


\title{
Comparative effect of the dose of pig manure used as pond fertilizer on the richness and distribution
} of phytoplankton taxa

\begin{abstract}
Objective : Pond pisciculture is characterized by a wide variety of fish farming practices, from the most extensive to the most intensive, including semi-intensive practices based on fertilization. Thus, the present work on phytoplankton culture was initiated to contribute to the improvement of fish production in fertilized ponds through better fertilizer management.

Methodology and results: The trial was conducted in 9 diversion ponds of the same area $(5.7 \times 5.7 \mathrm{~m})$ and depth $1 \mathrm{~m}$ at the aquaculture station of the Ferme d'Application et de Recherche (F.A.R) of the University of Dschang. A $1.5 \mathrm{~mm}$ mesh size net was attached to the inlet pipe of each pond to prevent fish intrusion from the reservoir. Each of the treatments consisting of $0 ; 800$ and $1000 \mathrm{~kg} / \mathrm{ha}$ of manure was applied in 3 ponds at random. The results showed that the species and genus richness of phytoplankton decreased with manure application, with 164 species recorded in the unfertilized ponds compared to 124 in the $800 \mathrm{~kg} / \mathrm{ha}$ manure treatment. Cyanophytes and Bacillariophytes were most abundant in unfertilized ponds while Euglenophytes and Pyrrophytes were dominant in ponds fertilized at the $1000 \mathrm{~kg}$ rate. Phytoplankton density increased with increasing amount of manure in the pond.

Conclusion and application: The rate of pig manure significantly influenced the phytoplankton richness and density in the pond. In sum, the $1000 \mathrm{~kg}$ rate can already be used if the optimal rate of phytoplankton production in ponds is sought in view of the progressive evolution of phytoplankton density with increasing rate. These results should contribute to the production of phytophagous fish, in particular the Carp in pond.
\end{abstract}

Keywords : Fertilization, pig manure, taxon, phytoplankton.

\section{INTRODUCTION}

L'aquaculture est en plein essor à travers la pisciculture et se pratique dans plusieurs infrastructures surtout les étangs. La pisciculture en étang s'appuie sur l'infrastructure d'élevage la plus anciennement connue et encore aujourd'hui la plus utilisée à travers le monde (Lazard, 2019). Elle se caractérise par une grande diversité de pratiques piscicoles des plus extensives aux plus intensives en passant par la pratique semi-intensive basée sur la fertilisation. Ainsi, pour mieux gérer les étangs dans un système semi-intensif et intensifier la production piscicole, il est nécessaire de connaître les organismes planctoniques qui les peuplent notamment le phytoplancton. Le phytoplancton (du grec phyton ou plante et planktos ou errant) est constitué par l'ensemble du plancton végétal, c'està-dire des microorganismes unicellulaires, coloniaux ou filamenteux photosynthétiques qui sont libres, passifs et en suspension dans la colonne d'eau. II est un des compartiments essentiels qui conditionne la chaîne alimentaire dans les écosystèmes aquatiques, surtout dans les étangs de pisciculture. II est l'un des aliments privilèges des poissons filtreurs en milieu naturel, tel que le tilapia qui est essentiellement phytoplanctonophage et consomme de multiples espèces de Chlorophycées, Cyanophycées, Euglenophycées. Les changements quantitatifs et qualitatifs qui ont lieu au sein des communautés phytoplanctoniques ont un impact sur l'ensemble de la chaîne trophique (Thyssen, 2008). Chikhaoui et al. (2008) ont révélé que la disponibilité des nutriments constitue le principal facteur qui contrôle le phytoplancton lorsque les conditions de lumière et de température sont adéquates. C'est ainsi que dans les étangs piscicoles les déjections des animaux notamment la fiente de poule, bouse de vache, crotte de lapin et lisier de porc sont apportés comme fertilisant afin d'assurer la disponibilité des nutriments nécessaires pour le développement du phytoplancton. Au Cameroun comme partout ailleurs, rares sont les travaux qui ont été effectués sur l'influence de ces fertilisants encore moins la dose sur le développement de la population phytoplanctonique. Pourtant plus de $80 \%$ de poisson produit sur le plan national provient des 
étangs fertilisés. C'est ainsi que ce travail a été initié avec pour objectif de contribuer à l'amélioration de la production de poisson en étangs fertilisés à travers une meilleure gestion des fertilisants. Plus

\section{MATERIEL ET METHODES}

Zone et période de l'étude : L'étude a été réalisée entre mai et novembre à la station aquacole de la Ferme d'Application et de Recherche (F.A.R) de l'Université de Dschang (LN : $5^{\circ} 44^{\prime}-5^{\circ} 36^{\prime}$ et LE : $10^{\circ} 06^{\prime}-9^{\circ} 85^{\prime}$, altitude : $1392-1396 \mathrm{~m}$ ) dans la zone agro-écologique soudano-guinéenne caractérisée par une courte saison sèche (mi- Novembre à mi-Mars) et une longue saison des pluies (mi- Mars à mi- Novembre). Les précipitations annuelles varient entre 1500 et $2000 \mathrm{~mm}$ et les températures oscillent entre $14^{\circ} \mathrm{C}$ (Mars-Octobre) et $25^{\circ} \mathrm{C}$ (Novembre-Février).

Obtention et conditionnement du lisier de porc : Le lisier (matière solide + urine) utilisé provenait de l'élevage des porcs nourris à l'aide d'un aliment composé (provende) fabriqué à base des produits et sous-produits locaux (maïs, remoulage, tourteaux de soja, de palmiste et de coton, son de riz, farine de sang, coquillage et concentré $5 \%$,). Hebdomadairement, le lisier a été collecté dans une porcherie le jour précédant son utilisation en étang, afin d'éviter la fermentation et la libération de l'azote. Les valeurs moyennes des caractéristiques du lisier analysées sont les suivantes : matière sèche $(30,5 \pm 2,21 \%)$, azote total $(33 \pm 1,11 \%)$, phosphore total $(9 \pm 2,30 \%)$.

Etangs expérimentaux : L'essai a été conduit dans 9 étangs en dérivation de même superficie $(5,7 \times 5,7 \mathrm{~m})$ et de profondeur $1 \mathrm{~m}$ avec un débit de 3,23 litre / minute, alimentés à partir d'un lac de retenu situé à $100 \mathrm{~m}$. Afin d'éliminer les organismes non souhaités (poissons) et d'augmenter l'alcalinité du milieu, les étangs ont été mis en asses pendant une période de 7 jours et chaulés à la chaux vive à une dose de $400 \mathrm{~kg} / \mathrm{ha}$. Un filet de $1,5 \mathrm{~mm}$ d'ouverture de maille a été fixé sur le tuyau d'alimentation de chaque étang en vue d'éviter l'intrusion des poissons venant de la retenue. Chacune des trois doses (correspondant aux traitements) constituées de: $0 ; 800$ et $1000 \mathrm{~kg} / \mathrm{ha}$ de lisier de porc a été appliquée dans un des étangs de façon aléatoire. Chaque traitement a été répété trois fois.

\section{Conduite de l'essai et collecte des données}

Fertilisation des étangs: En vue d'apporter les nutriments nécessaires à la production phytoplanctonique, le lisier de porc a été appliqué dans les étangs par épandage une fois par semaine pendant spécifiquement il s'agit d'évaluer l'effet de la dose de lisier de porc sur la composition, la répartition, la fréquence et la densité des taxons de phytoplancton en étangs.

une période de 6 mois. Les quantités de lisier ont été chaque fois pesées avant usage à l'aide de la balance électronique de précision $0,1 \mathrm{~g}$.

\section{Echantillonnage des organismes}

phytoplanctoniques: L'échantillonnage du phytoplancton a été effectué de façon bimensuelle. Le prélèvement a été réalisé en vingt points différents de la colonne d'eau de chaque étang à l'aide d'un récipient en polyéthylène étalonné de contenance 1 litre, soit un volume total de 20 litres/étang filtré au moyen d'un tamis à plancton de $40 \mu \mathrm{m}$ de maille. Un volume de $350 \mathrm{ml}$ concentré de phytoplancton a été récupéré, fixé par ajout de formol à $5 \%$ et conservé dans les bouteilles plastiques pour les analyses quantitatives et qualitatives.

Détermination des caractéristiques physicochimiques de l'eau: La transparence au disque de secchi, température, $\mathrm{pH}$, oxygène dissous et conductivité électrique ont été directement mesurée sur le terrain à l'aide respectivement d'un disque de secchi lesté et rattaché à une corde graduée, ThermoConductimètre, Thermo $\mathrm{pH}$-mètre, $\mathrm{pH}$ mètre, ThermoOxymètre et Thermo-Conductimètre de marque HANNA Les dosages de nitrite, nitrate et phosphates totaux ont été effectués par spectrophotométrie (spectrophotomètre HACH DR/2000) selon les techniques Alpha (Greenberg, 1985).

Analyse qualitative et quantitative du phytoplancton : L'analyse qualitative du phytoplancton a été effectuée selon Nguetsop et al. (2009). Ainsi, chaque sous-échantillon $(350 \mathrm{ml})$ préalablement filtré a été laissé au repos pendant 24 heures au minimum pour permettre la sédimentation du phytoplancton. Un volume de $300 \mathrm{ml}$ d'eau a été ensuite enlevé au-dessus du résidu phytoplanctonique par siphonage et les échantillons concentrés de phytoplancton ont été conservés dans des béchers. Après homogénéisation deux gouttes $(10 \mu l)$ de chaque échantillon concentré de phytoplancton ont été prélevées par une micropipette, montées entre lame et lamelle et observées à l'aide d'un microscope optique (objectifs $\times 40$ ) de marque Olympus, modèle $\mathrm{BH}-2$, équipé d'une lentille nomarski. Pour chaque échantillon, trois lames ont été préparées afin de s'assurer de la reproductibilité des lames (Nguetsop et al., 2009). Les identifications ont été faites en utilisant les 
clés de Bourelly et Manguin (1952); Couté et Rousselin (1975), Compère $(1976,1977)$; Iltis (1980) ; Gasse (1986); Krammer et Lange-Bertalot (1991). Les dénombrements cellulaires ont été effectués au microscope inversé, de marque ZEISS 471202 à l'objectif $\times 40$ tels que recommandés par Lund et al. (1958). Après homogénéisation, $10 \mathrm{ml}$ de l'échantillon ont été prélevés à l'aide d'une pipette étalonnée, déposé dans une coupelle de décantation et laissé sédimenter pendant 10 minutes. Des comptages ont été faits sur six champs pris aléatoirement dans la coupelle. Le

\section{RESULTATS}

Richesse des taxons phytoplanctoniques en fonction de la dose du lisier de porc : La richesse des taxons phytoplanctoniques résumé dans le tableau 1 fait apparaitre un total de 244 espèces de phytoplancton regroupées en 31 familles et 5 embranchements indépendamment de la dose. La richesse des espèces montre que la valeur la plus élevée (164 espèces) est enregistrée dans les étangs non fertilisés (témoin) et la plus faible (124 espèces) dans le traitement à la dose de $800 \mathrm{~kg} / \mathrm{ha}$ de lisier. En ce qui concerne la richesse générique, elle a diminué avec l'augmentation de la dose de lisier de porc. Quant à la répartition, 6 genres et 8 espèces de phytoplancton appartenant aux familles de Merismopediaceae, Gomphosphaeriaceae, Oocystaceae, Chlamidomonaceae, Ulotrichaceae et Eunotiaceae ont été répertoriés uniquement dans les minimum des unités (cellule, filament, cénobe ou colonie) dénombrées par répliquât d'un échantillon a été fixé à 400 , afin d'avoir une exactitude de \pm 10 à $95 \%$ d'intervalle de confiance.

Analyses statistiques : Les données collectées ont été soumises à l'analyse de la variance à un facteur (ANOVA 1). En cas de différences significatives entre les moyennes, le test de Duncan a été appliqué pour les séparer au seuil de signification $5 \%$. Le logiciel SPSS 20.0 (Statistical Package for Social Sciences) a été utilisé pour ces analyses.

étangs non fertilisés. En milieu fertilisé, 2 genres et 2 espèces de la famille de Gloeotrichiaceae et Oedogoniaceae ont été identifiés particulièrement dans les étangs recevant $800 \mathrm{~kg}$ de lisier alors que 4 genres et 6 espèces appartenant à la famille de Notocaceae, Aphanizomenonaceae, Orthoseiraceae et Peridiniaceae sont enregistrés dans les étangs fertilisés à la dose de $1000 \mathrm{~kg}$. Un total de 15 familles notamment Pseudanabaenaceae, Tolypothrichaceae, Nostocaceae, Oscillatoriaceae, Hydrodictyaceae, Scenedesmaceae, Mesotaeniaceae, Desmidiaceae, Zygnemataceae, Naviculaceae, Fragilariaceae, Surirellaceae, Melosiraceae, Aulacoseiraceae et Euglenaceae a été représenté transversalement dans tous les étangs quelle que soit la dose de lisier.

Tableau 1 : Richesse des taxons phytoplanctoniques

\begin{tabular}{|c|c|c|c|c|c|c|c|}
\hline \multirow[t]{2}{*}{ Embranchement } & \multirow[t]{2}{*}{ Familles } & \multicolumn{3}{|c|}{ Nombre de genres } & \multicolumn{3}{|c|}{ Nombre d'espèces } \\
\hline & & $0 \mathrm{~kg}$ & $800 \mathrm{~kg}$ & $1000 \mathrm{~kg}$ & Okg & $800 \mathrm{~kg}$ & $1000 \mathrm{~kg}$ \\
\hline \multirow{16}{*}{ Cyanophytes } & Notocaceae & 0 & 0 & 1 & 0 & 0 & 1 \\
\hline & Pseudanabaenaceae & 1 & 1 & 1 & 1 & 1 & 1 \\
\hline & Gloeotrichiaceae & 0 & 1 & 0 & 0 & 1 & 0 \\
\hline & Aphanizomenonaceae & 0 & 0 & 1 & 0 & 0 & 1 \\
\hline & Merismopediaceae & 1 & 0 & 0 & 1 & 0 & 0 \\
\hline & Tolypothrichaceae & 2 & 1 & 1 & 2 & 1 & 1 \\
\hline & Nostocaceae & 1 & 1 & 0 & 1 & 1 & 0 \\
\hline & Oscillatoriaceae & 2 & 2 & 2 & 2 & 4 & 4 \\
\hline & Gomphosphaeriaceae & 1 & 0 & 0 & 1 & 0 & 0 \\
\hline & Hydrodictyaceae & 1 & 1 & 1 & 1 & 1 & 1 \\
\hline & Oocystaceae & 1 & 0 & 0 & 1 & 0 & 0 \\
\hline & Micratiniaceae & 0 & 1 & 1 & 0 & 1 & 1 \\
\hline & Oedogoniaceae & 0 & 1 & 0 & & 1 & 0 \\
\hline & Chlamidomonaceae & 1 & 0 & 0 & 1 & 0 & 0 \\
\hline & Scenedesmaceae & 1 & 1 & 1 & 5 & 1 & 3 \\
\hline & Ulotrichaceae & 1 & 0 & 0 & 2 & 0 & 0 \\
\hline
\end{tabular}


Nana et al., J. Appl. Biosci. 2020 Effet comparé de la dose de lisier de porc utilisé comme fertilisant en étang sur la richesse et la distribution des taxons phytoplanctoniques

\begin{tabular}{|l|l|l|l|l|l|l|l|}
\hline Chlorophytes & Mesotaeniaceae & 1 & 2 & 1 & 1 & 2 & 1 \\
\cline { 2 - 8 } & Desmidiaceae & 8 & 8 & 6 & 46 & 35 & 28 \\
\cline { 2 - 8 } & Zygnemataceae & 3 & 3 & 3 & 16 & 19 & 19 \\
\hline \multirow{5}{*}{ Bacillariophytes } & Naviculaceae & 8 & 6 & 6 & 36 & 25 & 27 \\
\cline { 2 - 8 } & Fragilariaceae & 1 & 1 & 2 & 3 & 4 & 4 \\
\cline { 2 - 8 } & Epithemiaceae & 1 & 1 & 0 & 5 & 1 & 0 \\
\cline { 2 - 8 } & Eunotiaceae & 1 & 0 & 0 & 2 & 0 & 0 \\
\cline { 2 - 8 } & Surirellaceae & 1 & 1 & 1 & 8 & 2 & 2 \\
\cline { 2 - 8 } & Melosiraceae & 1 & 1 & 1 & 3 & 1 & 3 \\
\cline { 2 - 8 } & Aulacoseiraceae & 1 & 1 & 1 & 2 & 1 & 3 \\
\cline { 2 - 8 } & Nitzchiaceae & 0 & 1 & 1 & 0 & 1 & 1 \\
\cline { 2 - 8 } & Orthoseiraceae & 0 & 0 & 1 & 0 & 0 & 1 \\
\hline Euglenophytes & Euglenaceae & 4 & 4 & 5 & 22 & 19 & 24 \\
\cline { 2 - 7 } & Astasiaceae & 1 & 1 & 0 & 2 & 1 & 0 \\
\cline { 2 - 7 } & Colaciaceae & 0 & 0 & 1 & 0 & 0 & 1 \\
\hline \multirow{5}{*}{ Pyrrophytes } & Peridiniaceae & 0 & 0 & 1 & 0 & 0 & 3 \\
\hline
\end{tabular}

Influence de la dose de lisier de porc sur l'abondance relative des genres et espèces par embranchements phytoplanctoniques : L'abondance relative des genres et espèces en fonction de la dose de lisier de porc résumée dans le tableau 2 montre que les Chlorophytes ont été les plus représentés en termes de genres et espèces suivi des Bacillariophytes quelle que soit la dose de lisier considérée. Les Pyrrophytes ont été les plus faiblement représentés. Quel que soit l'embranchement, l'abondance relative des genres et espèces a faiblement varié en fonction de la dose de lisier de porc. Les Cyanophytes et Bacillariophytes ont été le plus abondant dans les étangs non fertilisés alors que les Euglenophytes et les Pyrrophytes ont été dominant dans les étangs fertilisés à la dose de $1000 \mathrm{~kg}$.

Tableau 2 : Abondance relative des embranchements en fonction de la dose de lisier de porc

\begin{tabular}{|l|l|l|l|l|l|l|}
\hline \multirow{2}{*}{ Embranchement } & Proportion des genres (\%) & Proportion des d'espèces (\%) \\
\cline { 2 - 7 } & $\mathbf{0 k g}$ & $\mathbf{8 0 0 k g}$ & $\mathbf{1 0 0 0} \mathbf{k g}$ & $\mathbf{0 k g}$ & $\mathbf{8 0 0} \mathbf{k g}$ & $\mathbf{1 0 0 0 k g}$ \\
\hline Cyanophytes & 13,55 & 10,16 & 10,16 & 03,27 & 03,27 & 03,27 \\
\hline Chlorophytes & 28,81 & 28,81 & 22,03 & 29,99 & 24,59 & 21,72 \\
\hline Bacillariophytes & 23,72 & 20,33 & 22,03 & 24,18 & 14,34 & 16,80 \\
\hline Euglenophytes & 08,47 & 08,47 & 10,16 & 9,83 & 8,16 & 10,24 \\
\hline Pyrrophytes & 0,00 & 0,00 & 01,69 & 0,00 & 0,00 & 1,22 \\
\hline Total 1 & 74,57 & 67,79 & 66,10 & 67,21 & 50,40 & 53,27 \\
\hline Total 2 & \multicolumn{7}{|c|}{$100(59)$} \\
\hline
\end{tabular}

$(\ldots)=$ Richesse totale

Effets de la dose de lisier de porc sur la fréquence des espèces phytoplanctoniques: L'influence de la dose de lisier de porc sur la fréquence des espèces est illustrée par la figure 1. II en ressort que de manière générale le genre Spirogyra a été le plus dominant aussi bien en milieu non fertilisé que celui fertilisé. Quelle que soit la dose de lisier l'espèce Spirogyra corrugata a été la plus fréquente. Nonobstant le genre Spirogyra, Micrasterias radians a été plus fréquent en étang non fertilisé et celui ayant reçu $800 \mathrm{~kg}$ de lisier de porc. 

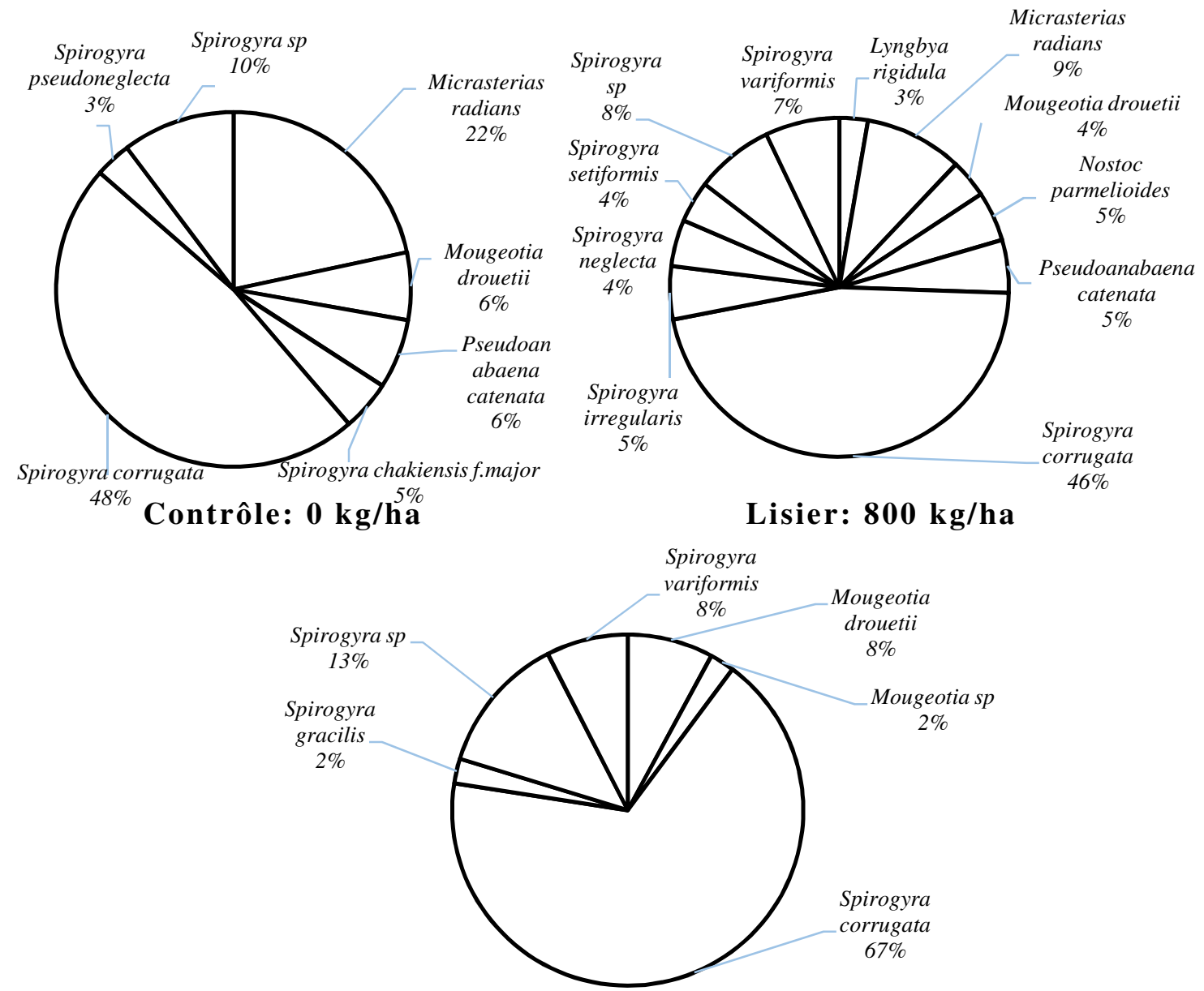

Lisier: $1000 \mathrm{~kg} / \mathrm{ha}$

Figure 1 : Fréquence des espèces phytoplanctoniques en fonction de la dose de lisier de porc

Densité phytoplanctonique : L'évolution de la densité du phytoplancton en fonction de la dose de lisier de porc est illustrée par la figure 2. II en ressort que la tendance, le profil et l'allure de l'évolution de la densité ont été en général comparable entre les doses. Toutefois, l'évolution a été de manière irrégulière avec la présence des pics de production. Quelle que soit la période de l'essai les valeurs les plus faibles de la densité phytoplanctonique sont observées dans le traitement témoin et les plus élevées dans les étangs fertilisés avec la plus grande dose de lisier de porc (1000 kg/ha). 


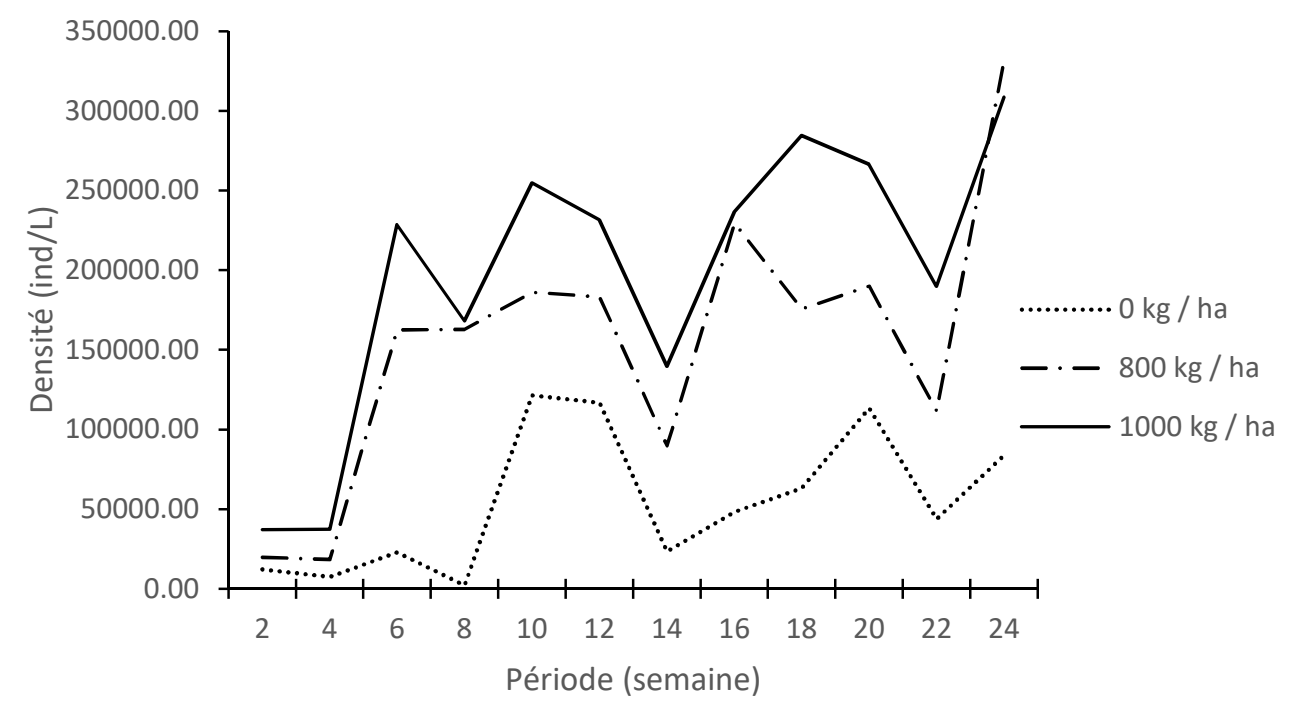

Figure 2 : Evolution de la densité phytoplanctonique en fonction de la dose de lisier de porc

$\begin{array}{lll}\text { Corrélations entre caractéristiques } & \text { l'oxygène dissous, les nitrites et nitrates dans le } \\ \text { physicochimiques de l'eau et la densité du } & \text { traitement témoin. A l'inverse la densité } \\ \text { phytoplancton en fonction de la dose de lisier de } & \text { phytoplanctonique est positivement corrélée au pH, } \\ \text { porc: Les corrélations entre caractéristiques } & \text { nitrates et phosphates dans les étangs fertilisés. La } \\ \text { physicochimiques de l'eau et la densité } & \text { densité phytoplanctonique a été négativement corrélée } \\ \text { phytoplanctonique est négativement et fortement } & \text { à la température et la transparence dans les étangs } \\ \text { corrélée }(p<0,01) \text { à la conductivité électrique, au pH, } & \text { fertilisés par contre positive en étang non fertilisé. }\end{array}$

Tableau 3 : Corrélation entre caractéristiques physicochimiques de l'eau et la densité du phytoplancton en fonction de la dose de lisier de porc

\begin{tabular}{|l|c|c|c|}
\hline $\begin{array}{l}\text { Caractéristiques } \\
\text { physicochimiques de } \\
\text { l'eau }\end{array}$ & \multicolumn{3}{|c|}{ Densité du phytoplancton } \\
\cline { 2 - 4 } & $\mathbf{0} \mathbf{k g}$ & $\mathbf{8 0 0 k g}$ & $\mathbf{1 0 0 0 k g}$ \\
\hline Transparence & $+0,061$ & $-0,494$ & $-0,494$ \\
\hline Température & $+0,760^{* *}$ & $-0,449$ & $-0,449$ \\
\hline $\mathbf{O}_{2}$ & $-0,994^{* *}$ & $-0,221$ & $-0,221$ \\
\hline $\mathbf{p H}$ & $-0,750^{* *}$ & $+0,482$ & $+0,482$ \\
\hline $\mathrm{NO}_{2}{ }^{-}$ & $-0,927^{* *}$ & $-0,541$ & $-0,541$ \\
\hline $\mathrm{NO}_{3}{ }^{-}$ & $-0,818^{* *}$ & $+0,091$ & $+0,091$ \\
\hline $\mathrm{PO}_{4}{ }^{-*}$ & $+0,482$ & $+0,202$ & $+0,202$ \\
\hline
\end{tabular}

${ }^{* *}$ : corrélation significative $p<0.01$ (bilatéral)

\section{DISCUSSION}

La composition et la structure des peuplements des micro-organismes aquatiques notamment le phytoplancton sont directement liées aux conditions environnementales. Ainsi, les résultats relatifs à l'effet de la dose de lisier de porc sur la composition du peuplement phytoplanctonique révèle que la richesse des espèces et genres phytoplanctoniques a été la plus élevée dans le traitement témoin. Cette observation est certainement due aux caractéristiques physicochimiques de l'eau influencées par la dose de lisier. En effet, Moss (1988) suggère quant à lui que seules les espèces capables de s'adapter à un fort enrichissement du milieu en nutriments et à un milieu présentant des conditions dites extrêmes suivant la physico-chimie, auront un avantage en condition eutrophe ; il en résulte ainsi une distribution inégale des 
individus des taxas. La population des taxas incapables de s'adapter décroit et celle des taxas pouvant s'adapter croit, et les proportions des taxas en fonction du niveau trophique sont ainsi modifiées suivant les populations de ces taxas. Les familles telles que Pseudanabaenaceae, Tolypothrichaceae, Nostocaceae, Oscillatoriaceae, Hydrodictyaceae, Scenedesmaceae, Mesotaeniaceae, Desmidiaceae, Zygnemataceae, Naviculaceae, Fragilariaceae, Surirellaceae, Melosiraceae, Aulacoseiraceae et Euglenaceae représentées transversalement dans tous les étangs s'expliquerait par le fait que ces familles sont généralement rencontrées dans des conditions écologiques variées, de préférence dans les eaux stagnantes de conductivité moyenne ou élevée tels que rapportés par Krammer et LangeBertalot (1986); Bourrelly et Manguin (1952). Les familles de Merismopediaceae, Gomphosphaeriaceae, Chlamidomonaceae et Ulotrichaceae étaient représentées spécifiquement dans le traitement témoin. En effet, ces familles sont généralement rencontrées dans les milieux spécifiques des eaux oligotrophes à mésotrophes avec une minéralisation modérée (Kaketa, 1996 ; Dodds et al., 1995). En outre ces familles ont des cellules à membrane épaisse, à contenu cellulaire homogène et très clair, capables de fixer l'azote atmosphérique (Rolland, 2009). Les familles de Gloeotrichiaceae, Notocaceae, Aphanizomenonaceae, Colaciaceae exclusivement représentées dans les étangs à lisier de porc s'expliqueraient par leur préférence écologique liée à la richesse en éléments nutritifs des eaux fertilisées, ainsi qu'aux types de prédateurs notamment les zooplanctons rencontrés dans ces traitements. La famille d'Oscillatoriaceae a été la plus diversifiée notamment dans les traitements aux doses de 800 et $1000 \mathrm{~kg} / \mathrm{ha}$ de lisier de porc. Ceci serait due au fait que les espèces appartenant aux genres Oscillatoria et Anabaena sont très compétitives grâce à leur capacité à secréter des cyanotoxines de différents types (Affsa/Afsset, 2006) qui inhibent la croissance des autres groupes d'algues et éliminent leurs prédateurs comme le zooplancton (Lavoie et al., 2007). Le genre Anabaena particulièrement représenté en étang fertilisé au lisier de porc à la dose $1000 \mathrm{~kg}$ serait dû à la capacité de ce dernier à s'adapter au milieu eutrophe. En effet, il est caractéristique des eaux riches en éléments nutritifs présentant son optimum de croissance dans les eaux fortement eutrophies (Descy et Willems, 1991). Les embranchements de phytoplancton tels que les Cyanophytes, Chlorophytes, Euglénophytes et Bacillariophytes identifiés ont été similaires à ceux observés par plusieurs auteurs (Njine et al., 2007 ; Nguetsop et al., 2009 ; Ponce-Palafox et al., 2010, Adjahouinou et al., 2012) en eau douce. Cette observation peut être justifiée par le caractère cosmopolite de ces embranchements et d'ailleurs très abondants dans les eaux douces africaines (Iltis, 1980). La fréquence spécifique des genres notamment celle de Spirogyra a été la plus élevée dans le traitement à la dose de $1000 \mathrm{~kg}$. Ce résultat corrobore ceux rapportés par Bonnard et al. (2003) ; Nguetsop (1990) ; Folefack (1989) qui ont trouvé les spirogyres en abondance dans les eaux de forte concentration en matière organique. L'abondance relative spécifique des embranchements de Cyanophytes, ainsi que des Euglenophytes a été plus élevée dans le traitement à la dose de $1000 \mathrm{~kg} / \mathrm{ha}$ de lisier de porc. Briand (2001) explique que les Cyanophytes supportent l'abondance de matière organique et de basses teneurs en oxygène dissous ce qui pourrait justifier la grande diversité des espèces de Cyanophytes. Selon Radji et al. (2013) la dominance des Euglénophytes serait due à la richesse en substances organiques putrescibles des eaux. Cette hypothèse s'explique dans notre cas par l'apport périodique de lisier de porc en étang dont la matière organique est décomposée par les micro-organismes. Les espèces de l'embranchement de Chlorophytes ainsi que celui de Bacillariophytes ont été plus abondants dans le traitement témoin et celles de l'embranchement des Euglenophytes dans le traitement à la dose de 1000 $\mathrm{kg} / \mathrm{ha}$ de lisier de porc. La proportion élevée des espèces appartenant à l'embranchement des Euglenophytes serait liée au fait qu'elles sont peu consommées par le zooplancton à cause de la qualité nutritionnelle faible que celle des Chlorophytes et Bacillariophytes (Lam et Silvester, 1979). La densité du phytoplancton a montré que le nombre d'individus par unité de volume obtenu a été significativement plus élevé dans les étangs fertilisés. Cette observation serait due à la richesse du milieu en éléments nutritifs issus de la décomposition de lisier de porc. D'ailleurs, les corrélations ont montré que, la densité phytoplanctonique a été hautement et significativement $(p<0,01)$ négative entre la conductivité électrique, le pH, l'oxygène dissous, les nitrites et les nitrates dans le traitement témoin. Les Pyrrophytes très peu diversifiés n'ont été présents que dans les étangs fertilisés. Ceci serait dû au fait que ces embranchements sont le plus souvent des algues marines et leur présence dans les eaux douces se limite à une trentaine de genres peu fréquents et donc n'existent qu'à l'état de rareté dans les eaux soudaniennes (lltis, 1980). 


\section{CONCLUSION ET APPLICATION DES RESULTATS}

Les résultats de la dose de lisier de porc utilisé comme fertilisant en étang sur la richesse et la distribution des taxons phytoplanctoniques ont montré une influence sur toutes les caractéristiques testées notamment la composition, la répartition, la fréquence et la densité des taxons de phytoplancton en étangs. En effet, la richesse spécifique ainsi que générique ont diminué avec l'augmentation de la dose de lisier de porc. Par contre,

\section{REFERENCES BIBLIOGRAPHIQUES}

Adjahouinou D.C, Liady N.D, Fiogbe D.E. 2012. Diversité phytoplanctonique et niveau de pollution des eaux du collecteur de Dantokpa (Cotonou-Bénin) International Journal of Biological and Chemical Sciences. 6 (5) : 19381949.

Afssa/Afsset. 2006. Évaluation des risques liés à la présence de cyanobactéries et de leurs toxines dans les eaux destinées à l'alimentation, à la baignade et autres activités récréatives. Rapport commun sur les risques sanitaires liés à la présence de cyanobactéries dans l'eau, $232 p$.

Bonnard R, Lafont M, Pimpec L.E.P. 2003. Notions d'hydro-écologie et de qualité biologique des eaux courantes. Ingénieries. $33:$ 3-12.

Bourrelly P, Manguin E. 1952. Algues d'eau douce de la Guadeloupe et dépendances. SEDES. Paris, $276 \mathrm{p}$.

Briand J.F. 2001. Cyanobactéries toxiques : Prolifération et production toxinique de deux espèces de Cyanobactéries toxiques de la région parisienne. Thèse de Doctorat. Muséum National d'Histoire Naturelle, Paris, $270 \mathrm{p}$.

Chikhaoui M.A, Hlaili A.S \& Mabrouk H.H. 2008. Réponses saisonnières du phytoplancton aux rapports d'enrichissements $\mathrm{N}: \mathrm{Si}: \mathrm{P}$ dans la lagune de Bizerte (Sud-Ouest de la Méditerranée). Comptes Rendus Biologies, 331: $389-408$

Compère P. 1976. Algues de la région du lac Tchad. VChorophycophytes 1ere partie. Cah. O.R.S.T.O.M., ser. Hydrobiol. 10 (2) : 77-118.

Compère P. 1977. Algues de la région du lac Tchad. VIIChorophycophytes 3e partie : Desrnidiées. Cah. O.R.S.T.O.M., Hydrobiol. 11 (2) : 77-177.

Descy JP, Willems C. 1991. Contribution à la connaissance du phytoplancton de la Moselle (France). Cryptogamie, Algologie. 12 (2) : 87100. la densité a augmenté avec l'accroissement de la quantité de lisier en étang. Au regard de l'évolution progressive de la densité phytoplanctonique avec l'augmentation de la dose durant toute la phase de l'expérimentation, il est nécessaire de rechercher la dose optimale de production en étang. Ces données vont permettre à améliorer l'élevage des espèces de poisson phytophage notamment les carpes en étang.

Dodds W.K. Gudder D.A, Mollenhauer D. 1995. The Ecology of Nostoc. Journal of Phycology. 31: 218.

Folefackc A. 1989. Étude de la flore algale du lac municipal de Yaoundé. Mémoire de Maîtrise, Univ. Yaoundé, $77 \mathrm{p}$.

Gasse F. 1986. Diatom assemblages in East Africa: classification, distribution and ecology. Rev. Hydrobiol. Trop. 16, 3-34.

Greenberg A. 1985. Standard methods for the examination of water and waste water. Édit. American Public Health Association, Washington, $16{ }^{\text {ème }}$ edition, $1268 p$.

Iltis A. 1980. Les algues. La Flore et Faune Aquatiques de l'Afrique Sahélo-Soudanienne (Tome I), Durand JR, Lévêque C (eds). Éditions O.R.S.T.O.M, Collection Initiations Doc. Tech. 44: $9-61$

Jérôme L. 2019. Piscicultures du monde aujourd'hui et demain ; collection des Livres de l'Académie d'agriculture de France. $263 p$

Kaketa A.G.S. 1996. Bacillariophycées, Desmidiées et Euglenophycées de la Région de Kisangani. (Zaire). Acad. Royale des Sciences D'OutreMer- (Mémoire), 23 (3) : 5-232.

Krammer k, Lange-Bertalot H. 1986. Süsswasserflora von Mitteleuropa. Bacillariophyceae. Teil 1: Naviculaceae. Fischer verlag, Stuttgart, Allemagne, $876 \mathrm{p}$.

Krammer k, Lange-Bertalot H. 1991. Süsswasserflora von Mitteleuropa. Bacillariophyceae. Teil 3: Centrales, Fragilariaceae, Eunotiaceae. Fischer verlag, Stuttgart, Allemagne, $576 \mathrm{p}$.

Lam C.W.Y, Silvester W.B. 1979. Growth interaction among blue-green algae. Hydrobiology. 63 (2) : 135-143.

Lavoie I, Laurion I, Warren A, Vincent W.F. 2007. Les fleurs d'eau de Cyanobactéries. Revue littéraire. INRS rapport $N^{\circ} 916,12 \mathrm{p}$. 
Lund J.W.G, Kilpling C, Le Cren E.D. 1958. The inverted microscope method of estimating algal numbers, and the statistical basis of estimation by counting. Hydrobiology. 11: 143-170.

Moss B. 1998. Ecology of fresh waters: man and medium, past to future. Blackwell Science, Oxford, $557 \mathrm{p}$.

Nguetsop V. F. 1990. Flore algale de quelques marécages à Cyrtosperma senegalense dans la région de Yaoundé. Mémoire de Maîtrise, Univ. Yaoundé, $88 \mathrm{p}$.

Nguetsop V.F. Fonkou T Lekeufack M et Pinta J.Y. 2009. Assemblages d'algues et relations Avec quelques paramètres environnementaux dans deux sites marécageux de l'Ouest-Cameroun. Revue des Sciences de l'Eau. 22 (1) 15-27.

Njine T, Kemka N, Zebaze Togouet S.H, Nola M, Niyitegeka D, Ayissi Etoundi P.T, Foto M.S. 2007. Peuplement phytoplanctonique et qualité des eaux en milieu lacustre anthropisé : cas du lac municipal de Yaoundé (Cameroun). African Journal of Science and Technology. Science and Engineering Series. 8 (1) : $39-51$.

Ponce-Palafox J.T, Arredondo-Figueroa J.L, CastilloVargasmachuca S, Rodríguez-Chávez G, Benítez-Valle A, Regalado-De Dios M, MedinaCarrillo F, Navarro-Villalobos R, GómezGurrola J. and López-Lugo P. 2010. The effect of chemical and organic fertilization on phytoplankton and fish production in carp (cyprinidae) polyculture system. Rev. Bioscienc. 1: 44-50.

Radji R, Bandje A, Issifou L, Edorh T, Kouami K. 2013. Diversité et dynamique des assemblages phytoplanctoniques dans les écosystèmes aquatiques au Sud du Togo. Afrique Science. 09 (2) : $67-77$

Roland A. 2009. Dynamique et diversité du phytoplancton dans le réservoir marne (bassin versant de la Seine). Thèse de Doctorat Université de Savoie, $261 \mathrm{p}$.

Thyssen M. 2008. Analyse à haute fréquence spatiale et temporelle du phytoplancton à l'aide de la cytométrie en flux automatisée et immergeable. Thèse de doctorat. pp.217. 